\title{
Synthesis and characterisation of a binder cement replacement based on alkali activation of fly ash waste
}

\begin{abstract}
This study considers the valorisation of an important amount of fly ash generated as waste in the process of coal burning in a thermal power plant of Iasi municipality (Romania). The waste valorisation is related to the development of a new building material by using the alkaline activated fly ash based on the fact that inorganic polymers with similar properties to ordinary Portland cement may be obtained by alkaline treatment. Three main operating parameters were considered for the synthesis of this material: the liquid/solid ratio, the concentration of alkaline solution and the activation temperature. By minimizing the activation temperature and recycling the hydroxide solution, the synthesis of low-cost materials for partial cement replacement in concrete was possible. The synthesized materials were thoroughly characterised for analysis of chemical composition, morphology, physical and mechanical properties by XRD, SEM, EDX, TGA, FTIR, density and compressive strength. According to experimental results, the synthesized materials have a bigger specific surface area as compared to that determined for fly ash. The XRD and FTIR analysis proved that fly ash waste contains: quartz, mullite, iron oxide and a vitreous phase, while the synthesized materials after the fly ash alkaline treatment contain: quartz, sodalite, chabazite, geopolymer gel.
\end{abstract}

Keyword: Alkaline treatment; Cement replacement; Chemical composition; Fly ash; Morphology; Physical and mechanical properties 